\title{
Logistic regression analysis of contrast-enhanced ultrasound and conventional ultrasound of follicular thyroid carcinoma and follicular adenoma
}

\author{
Qiong $\mathrm{Wu}^{\#} \wedge$, Yanhui Qü ${ }^{\#}$ Yi Li, Yilun Liu, Jian Shen, Yan Wang^ \\ Department of Ultrasound in Medicine, Affiliated Sixth People's Hospital of Shanghai Jiao Tong University, Shanghai Institute of Ultrasound in \\ Medicine, Shanghai, China \\ Contributions: (I) Conception and design: Y Wang; (II) Administrative support: Y Wang; (III) Provision of study materials or patients: Q Wu, Y Qu, \\ Y Li, Y Liu, J Shen; (IV) Collection and assembly of data: All authors; (V) Data analysis and interpretation: Q Wu, Y Qu; (VI) Manuscript writing: \\ All authors; (VII) Final approval of manuscript: All authors. \\ \#These authors contributed equally to this work. \\ Correspondence to: Yan Wang, MD. Department of Ultrasound in Medicine, Affiliated Sixth People's Hospital of Shanghai Jiao Tong University, \\ Shanghai Institute of Ultrasound in Medicine, 600 Yishan Road, Shanghai 200233, China. Email: yannanfly@126.com.
}

\begin{abstract}
Background: Follicular thyroid carcinoma (FTC) is prone to recurrence and hematogenous metastasis, preoperative accurate diagnosis is still needed to help clinicians select the best surgical methods to improve the prognosis of patients. The aim of this study was to find specific conventional ultrasound (US) and contrast-enhanced ultrasound (CEUS) characteristics of FTC and to explore their diagnostic value in the differential diagnosis between FTC and follicular adenoma (FA).

Methods: This retrospective study included 258 thyroid follicular neoplasms (172 FAs and 86 FTCs) in 244 consecutive patients who underwent preoperative conventional US, and 72 of them underwent both conventional US and CEUS. Sonograms were reviewed in consensus by two experienced radiologists for various conventional US and CEUS features. Multivariate logistic regression analysis was performed to determine independent risk factors for FTC.

Results: Independent risk factors of conventional US for FTC were heterogenicity (OR =7.477, 95\% CI: 2.848-19.629), unevenly thick halo (OR $=5.643,95 \%$ CI: 3.234-9.848) and calcifications (OR $=1.748$, 95\% CI: 1.098-2.783). While independent risk factors determined with the combination of CEUS and conventional US were unevenly thick halo (OR =5.770, 95\% CI: 1.310-25.409) and absent or irregular rim enhancement (OR $=27.000,95 \%$ CI: 2.445-298.178). The area under the receiver operating characteristic curve of the final two logistic regression models was 0.835 and 0.838 .

Conclusions: Conventional US is an efficient diagnostic tool in the differential diagnosis of FTC and FA to help clinicians in making appropriate decisions while CEUS failed to provide additional diagnostic value in the study, thus the value of CEUS remains to be verified by further studies with larger sample sizes.
\end{abstract}

Keywords: Follicular thyroid carcinoma (PTC); follicular adenoma; logistic regression; conventional ultrasound; contrast-enhanced ultrasound

Submitted Aug 08, 2021. Accepted for publication Sep 22, 2021.

doi: $10.21037 / \mathrm{gs}-21-535$

View this article at: https://dx.doi.org/10.21037/gs-21-535

\footnotetext{
^ ORCID: Qiong Wu, 0000-0002-2113-7714; Yan Wang, 0000-0003-1675-9065.
} 


\section{Introduction}

Thyroid cancer is the most common malignancy of the endocrine system, and it has risen significantly in recent years. The two most common histological subtypes are papillary thyroid carcinoma (PTC) and follicular thyroid carcinoma (FTC), which account for approximately 70-75\% and $15-20 \%$ of all thyroid malignancies, respectively (1). As is known, most PTC have good prognosis with slow growth, while FTC is prone to recurrence and hematogenous metastasis to distant locations such as bone and lung (2). Unfortunately, intraoperative frozen-section analysis is not reliable and does not provide useful information for intraoperative decision-making $(3,4)$. Although FTC can be confirmed postoperatively by histological observation of capsular or vascular invasion, preoperative accurate diagnosis is still needed to help clinicians select the best surgical methods to improve the prognosis of patients.

For now, the main preoperative diagnostic methods for thyroid cancer are fine needle aspiration cytology and conventional ultrasound (US). Ultrasonography (US)guided fine needle aspiration (FNA) cytology has become the most accurate modality for preoperative definitive assessment of thyroid nodules. Follicular adenoma (FA) and FTC are thyroid neoplasms consisting of differentiated follicular cells with similar cytomorphological features (5-7). In several previous studies, when a FNA sample of a thyroid nodule reveals a follicular neoplasm, approximately $80-90 \%$ of such lesions are benign (8-10). However, most researchers still believe that thyroid malignancy cannot easily be determined in follicular neoplasms $(11,12)$. Conventional ultrasound is the main imaging tool in the characterization of thyroid nodules, and could help clinicians determine when a nodule requires FNA or sonographic follow-up. Several sonographic features including hypoechogenicity, irregular margin, taller-thanwide shape, and microcalcification are associated with malignancy especially in PTC, but none were proved to be appropriate for FTC (13-15). Koike's prospective research reported that the sensitivity of the classic US characteristics of thyroid carcinoma was found to be $86.5 \%$ for non-follicular neoplasms, but only $18.2 \%$ for follicular carcinomas (16).

Contrast-enhanced ultrasound (CEUS) has been widely used for assessment of thyroid-related diseases, relying on differentiating background tissues acoustic response (linear) from the non-linear response of bubbles. Through observation of the microcirculation perfusion within the lesion, researchers have tried to distinguish benign from malignant thyroid nodules with specific contrast-enhanced features (17-20). However, to the best of our knowledge, there are no studies focusing on the diagnostic value of the contrast-enhanced features in the differentiation of FTC and FA before. Furthermore, a few studies have tried to find the sonographic difference between them, but no agreement has yet been reached (21-24). In the present study, we aimed to find specific conventional US and CEUS characteristics of FTC and to explore their diagnostic value in the differential diagnosis between FTC and FA. We present the following article in accordance with the STARD reporting checklist (available at https://dx.doi.org/10.21037/ gs-21-535).

\section{Methods}

\section{Patients}

We retrospectively reviewed medical records of 303 thyroid follicular neoplasms (including a histopathologic diagnosis of follicular carcinoma or follicular adenoma) which underwent conventional US before final surgery in our institution from January 2013 to August 2019. Of these nodules, 45 were excluded due to US images of unsatisfactory quality. In the end, a total of 258 thyroid follicular neoplasms in 244 consecutive patients were recruited for the study, and 72 cases of them underwent both conventional US and CEUS before surgery. Among them, there were 50 males and 194 females, aged 17-80 years, with an average age of $48.3 \pm 15.1$ years. The mean US-measured maximum diameter of the 258 nodules was $32.4 \pm 13.4 \mathrm{~mm}$ (range, $5.0-73 \mathrm{~mm}$ ). The study was conducted in accordance with the Declaration of Helsinki (as revised in 2013). The study was approved by the institutional review board of Shanghai Jiao Tong University Affiliated Sixth People's Hospital [No. 2018-KY-040 (K)] and individual consent for this retrospective analysis was waived.

\section{Conventional ultrasound}

All sonographic examinations were performed with color Doppler ultrasound diagnostic equipments such as Acuson S2000 (Siemens, Erlangen, Germany), Mylab 90 Xvision (ESAOTE, Italy), iU Elite (Philips Healthcare, Bothell, WA), and HI Vision Preirus (Hitachi Medical, Tokyo, Japan). Line array probes were used with frequencies from 6-15 MHz. The research subject was positioned at a supine position with necks hyperextended to fully 
expose the thyroid area. The following sonographic features were recorded for each nodule: maximum tumor diameter, echogenicity (hyperechoic, isoechoic, hypoechoic [with respect to the background thyroid), echo texture (homogeneous, heterogeneous), margin (regular, irregular), border (clear, obscure), extracapsular extension(absent, present), peripheral halo sign (absent, thin, unevenly thick), cystic changes (absent, present), range of cystic changes [absent, predominantly solid $(<50 \%)$, predominantly cystic $(\geq 50 \%)$ ], calcifications [absent, microcalcifications (calcification less than $2.0 \mathrm{~mm}$ in diameter), macrocalcifications or peripheral calcifications], the distribution patterns of nodule vascularity on color Doppler (predominantly intranodular vascularity, predominantly perinodular vascularity, diffuse or absent). Besides, we also recorded whether the peripheral halo in two-dimensional ultrasound was related to the observation of peripheral blood vessels in color Doppler.

\section{Contrast-enhanced ultrasound}

CEUS was performed with a linear probe of 3-9 $\mathrm{MHz}$ (L522) of ultrasound system (ESAOTE MyLab 90 Xvision, Italy) after Conventional US, and the sampling frame was adjusted to include the lesion and the surrounding normal tissue. As bubble disruption was strictly related to depth and focalization of a US beam, focus was placed deeper than the nodule being examined at low MI (0.05-0.07) to avoid the destruction of microbubbles. Sulphur hexafluoride (SonoVue ${ }^{\circledR}$, Bracco International, Milan, Italy) was injected with an intravenous bolus of $1.2 \mathrm{~mL}$ via a 20 -gauge cannula followed by a $10-\mathrm{mL}$ saline flush. In the meantime, the imaging timer on the US machine was started, and each contrast imaging acquisition lasted at least 120 s was stored in the machine hard disk. The following CEUS characteristics were recorded: arrival time (time from intravenous administration to appearance of the first bubble in tumor compared with that of surrounding parenchyma), classified as earlier, synchronous or later; entrance mode of microbubble echoes [centripetal, non-centripetal (diffuse, centrifugal)]; peak intensity (hypo-enhancement, iso-enhancement, or hyper-enhancement); homogeneity of enhancement pattern at peak (homogeneous, heterogeneous); early washout compared with the adjacent thyroid parenchyma(absent, present); wash-out style (rapid, slow); perfusion defects (absent, present); range of perfusion defects $(<50 \%, \geq 50 \%)$; rim enhancement (the rim enhancement pattern of the lesion should be thin and regular): classified as absent or irregular, present.

All the preoperative sonograms including conventional ultrasound and CEUS were reviewed in random order on the screen by two skilled radiologists ( $\mathrm{Q} \mathrm{Wu}$ and $\mathrm{Y} \mathrm{Qu}$ ) blinded to the clinical and pathological information. Each reader independently analyzed conventional US and CEUS images first, and then reviewed the cases with discrepancy in their initial evaluation together. Finally, a consensus was reached after discussion.

\section{Statistical analysis}

Statistical analysis was performed with a statistical package (SPSS 19.0, Chicago, IL, USA). Quantitative data were expressed as the mean and standard deviation, and groups were compared using Student's $t$-test. The Chi-square test or Fisher exact test was used to compare categorical data. Odds ratios (ORs) with $95 \%$ confidence intervals (CIs) were calculated using logistic regression. Only those factors found to be significantly associated with follicular carcinomas in the univariable analyses $(\mathrm{P}<0.05)$ were then included in the multivariable analysis. The variables that showed statistical correlation by logistic regression analysis were considered as independent predictive factors for follicular carcinomas. The receiver operating characteristic (ROC) analysis was performed to determine the diagnostic value of different logistic regression formulas. In all cases, two-tailed $\mathrm{P}$ values lower than 0.05 were considered to indicate statistical significance.

\section{Results}

Among all 258 thyroid follicular neoplasms, 172 were follicular adenomas and 86 were follicular thyroid carcinomas which consisted of 74 minimally invasive FTCs and 12 widely invasive FTCs, and widely invasive FTC is mainly recognized due to extensive invasion by histopathology, and there were no lymph node metastases in all the FTC cases. The clinical and conventional ultrasound features of follicular adenomas and carcinomas are shown in Table 1. There was a significant difference in sex between research subjects with follicular carcinoma (male to female ratio, 5:12) and those with follicular adenoma (male to female ratio, 25:134) $(\mathrm{P}<0.05)$, which indicated that male patients were more common in FTC group than FA group. Other variables, such as patients' age $(48.3 \pm 15.2$ versus $48.3 \pm 15.0$ years $)$, maximum tumor diameter $(34.4 \pm 14.3$ versus $31.4 \pm 12.9 \mathrm{~mm}$ ), did not show a significant difference 
Table 1 Clinical and conventional ultrasound features of FTC and FA

\begin{tabular}{|c|c|c|c|}
\hline Feature & FTC $(n=86)$ & FA $(n=172)$ & $P$ value \\
\hline Age (y) & $48.3 \pm 15.2$ & $48.3 \pm 15.0$ & 0.969 \\
\hline Sex & $\mathrm{n}=85$ & $n=159$ & \\
\hline Female & 60 & 134 & 0.012 \\
\hline Male & 25 & 25 & \\
\hline Maximum diameter (mm) & $34.4 \pm 14.3$ & $31.4 \pm 12.9$ & 0.092 \\
\hline Echogenicity & & & 0.007 \\
\hline Hypoechoic & 40 & 47 & \\
\hline Isoechoic & 45 & 120 & \\
\hline Hyperechoic & 1 & 5 & \\
\hline Echo texture & & & $<0.001$ \\
\hline Homogeneous & 7 & 83 & \\
\hline Heterogeneous & 79 & 89 & \\
\hline Margin & & & $<0.001$ \\
\hline Regular & 71 & 168 & \\
\hline Irregular & 15 & 4 & \\
\hline Border & & & 0.007 \\
\hline Clear & 79 & 170 & \\
\hline Obscure & 7 & 2 & \\
\hline Extracapsular extension & & & 0.004 \\
\hline Absent & 81 & 172 & \\
\hline Present & 5 & 0 & \\
\hline Peripheral halo sign & & & $<0.001$ \\
\hline Absent & 25 & 58 & \\
\hline Thin & 6 & 109 & \\
\hline Unevenly thick & 55 & 5 & \\
\hline Cystic changes & & & 0.012 \\
\hline Absent & 57 & 84 & \\
\hline Present & 29 & 88 & \\
\hline Range of cystic changes & & & 0.023 \\
\hline Absent & 57 & 84 & \\
\hline Predominantly solid $(<50 \%)$ & 26 & 74 & \\
\hline Predominantly cystic ( $\geq 50 \%)$ & 3 & 14 & \\
\hline Calcifications & & & 0.004 \\
\hline Absent & 55 & 140 & \\
\hline Microcalcifications & 7 & 12 & \\
\hline $\begin{array}{l}\text { Macrocalcifications or } \\
\text { peripheral calcifications }\end{array}$ & 24 & 20 & \\
\hline
\end{tabular}

Table 1 (continued)
Table 1 (continued)

\begin{tabular}{lccc}
\hline Feature & FTC $(n=86)$ & FA $(n=172)$ & P value \\
\hline Vascularity & 11 & 9 & 0.001 \\
$\begin{array}{l}\text { Predominantly intranodular } \\
\text { vascularity }\end{array}$ & & \\
$\begin{array}{l}\text { Predominantly perinodular } \\
\text { vascularity }\end{array}$ & 25 & 90 \\
Diffuse or absent & 50 & 73 \\
\hline
\end{tabular}

FTC, follicular thyroid carcinoma; FA, follicular adenoma.

between FTC and FA groups (all P>0.05).

\section{Conventional US}

On univariable analysis, sonographic features including echogenicity, echo texture, margin, border, extracapsular extension, peripheral halo sign (91 out of 114 FA cases with peripheral halo in two-dimensional ultrasound were found peripheral blood vessels in color Doppler, while 57 out of 61 were found in FTC), cystic changes, range of cystic changes, calcifications, and nodule vascularity showed significant difference between FA and FTC (all $\mathrm{P}<0.05$ ). These factors were thus included in the further multivariable analysis, which identified the following independent risk factors for follicular carcinoma: hypoechoic echogenicity (OR $=0.475,95 \%$ CI: $0.232-0.971$, as $\mathrm{OR}<1$ usually indicates that exposure is associated with lower odds of the outcome, hypoechoic echogenicity was excluded in the following study), heterogenicity ( $\mathrm{OR}=7.477,95 \% \mathrm{CI}: 2.848-19.629)$, unevenly thick halo (OR $=5.643,95 \%$ CI: 3.234-9.848) and calcifications $(\mathrm{OR}=1.748,95 \%$ CI: $1.098-2.783)$ (model 1) (Table 2). Then, the above three sonographic features were enrolled in the final multivariable logistic regression model of conventional US (model $1^{*}$ ). Figure 1 show the conventional US images of follicular adenomas and carcinomas, respectively.

\section{CEUS}

In our retrospective study, only 72 cases had undergone both conventional US and CEUS before the final surgery. The CEUS features of follicular adenomas and carcinomas are detailed in Table 3. Among the CEUS characteristics, early washout, rapid wash-out style, and absent or irregular rim enhancement showed significant associations with follicular carcinoma in the univariable analysis (all $\mathrm{P}<0.05$ ). 
Table 2 Multivariate logistic regression analysis

\begin{tabular}{lccc}
\hline Variable & P value & Odds ratio & $95 \% \mathrm{Cl}$ \\
\hline \multicolumn{3}{l}{ Multivariate logistic regression model of conventional US } \\
Hypoechoic echogenicity & 0.041 & 0.475 & $0.232-0.971$ \\
Heterogenicity & 0 & 7.477 & $2.848-19.629$ \\
$\begin{array}{l}\text { Absence of cystic } \\
\text { changes }\end{array}$ & 0.893 & 0.854 & $0.086-8.495$ \\
Irregular margin & 0.059 & 4.539 & $0.946-21.790$ \\
Obscure border & 0.05 & 9.426 & $1.004-88.464$ \\
Extracapsular extension & 0.999 & $\mathrm{NA}$ & $\mathrm{NA}$ \\
Unevenly thick halo & 0 & 5.643 & $3.234-9.848$ \\
Range of cystic changes & 0.932 & 1.091 & $0.147-8.103$ \\
Calcifications & 0.019 & 1.748 & $1.098-2.783$ \\
Vascularity & 0.232 & 1.257 & $0.864-1.830$ \\
Constant & 0 & 0.016 &
\end{tabular}

Multivariate logistic regression model of CEUS in combination with conventional US

\begin{tabular}{lccc} 
Hypoechoic echogenicity & 0.213 & 0.342 & $0.063-1.852$ \\
Heterogenicity & 0.998 & NA & NA \\
Unevenly thick halo & 0.02 & 5.77 & $1.310-25.409$ \\
Range of cystic changes & 0.306 & 2.496 & $0.432-14.412$ \\
Calcifications & 0.145 & 2.455 & $0.734-8.208$ \\
Vascularity & 0.494 & 0.741 & $0.313-1.750$ \\
Early washout & 0.453 & 0.505 & $0.085-3.001$ \\
Rapid washout & 0.193 & 4.11 & $0.490-34.495$ \\
Absent or irregular rim & 0.007 & 27 & $2.445-298.178$ \\
enhancement & & & \\
Constant & 0.998 & 0 & \\
\hline
\end{tabular}

US, conventional ultrasound; CEUS, contrast-enhanced ultrasound; $\mathrm{Cl}$, confidence intervals; NA, not available.

We then put the aforementioned significant CEUS features into the further multivariable analysis along with potential sonographic features (echogenicity, echo texture, peripheral halo sign, range of cystic changes, calcifications, nodule vascularity). Among all of the characteristics, independent risk factors for follicular carcinoma were: unevenly thick halo (OR $=5.770,95 \%$ CI: $1.310-25.409)$ and absent or irregular rim enhancement (OR $=27.000$, 95\% CI: $2.445-$ 298.178) (model 2) (Table 2). Then, these two factors were enrolled in the final multivariable logistic regression model of CEUS in combination with conventional US (model $2^{*}$ ). Figure 2 show the conventional gray-scale and contrastenhanced images of follicular adenomas and carcinomas, respectively.

\section{Diagnostic performance}

For the differential diagnosis between follicular adenomas and carcinomas, the sensitivity, specificity, positive predictive value (PPV) and negative predictive value (NPV) of individual ultrasound features are summarized in Table 4. In the multivariable logistic regression model of conventional US, unevenly thick halo had the highest diagnostic accuracy $(86.0 \%)$, with a sensitivity of $64.0 \%$, specificity of $97.1 \%$, PPV of $91.7 \%$ and NPV of $84.3 \%$. While in the logistic regression model of CEUS in combination with conventional US, unevenly thick halo also had the highest diagnostic accuracy $(86.1 \%)$, with a sensitivity of $68.0 \%$, specificity of $95.7 \%$, PPV of $89.5 \%$ and NPV of $84.9 \%$. The absent or irregular rim enhancement showed a very high sensitivity (96\%) and NPV (96\%), which suggested a useful feature for ruling out follicular thyroid cancer. We further conducted ROC analysis for the final two logistic regression models to define the preoperative diagnostic value of these factors in differentiating FTC from FA. The AUC (area under the curve) of model $1^{*}$ was 0.835 , and the $95 \%$ CI was $0.782-0.889$, while the AUC of model $2^{*}$ was 0.838 , and the $95 \%$ CI was $0.734-0.942$ (Figure 3).

\section{Discussion}

Previous studies have suggested that thyroid follicular neoplasms are more common in female, but the incidence of follicular carcinoma in male is higher than that in thyroid adenomas, and a follicular carcinoma diagnosis is said to be more often present in a larger tumor of an older patient $(21,25)$. In the present research, we found that male patients were more common in FTC group than FA group $(\mathrm{P}<0.05)$. While older age ( $48.3 \pm 15.2$ versus $48.3 \pm 15.0$ years) and larger tumor size $(34.4 \pm 14.3$ versus $31.4 \pm 12.9 \mathrm{~mm})$ were shown in follicular carcinoma group although there was no statistically significant difference (all $\mathrm{P}>0.05$ ), in accordance with both Zhang and Kuo's studies $(23,24)$. With relatively large sample size, we remain skeptical about the distribution difference of age and tumor size between FTC and FA, and further multicenter studies are necessary to validate these findings.

Heterogenicity has been reported to be associated with follicular carcinoma $(\mathrm{OR}=24.881 ; \mathrm{P}<0.01)(23)$. Some 

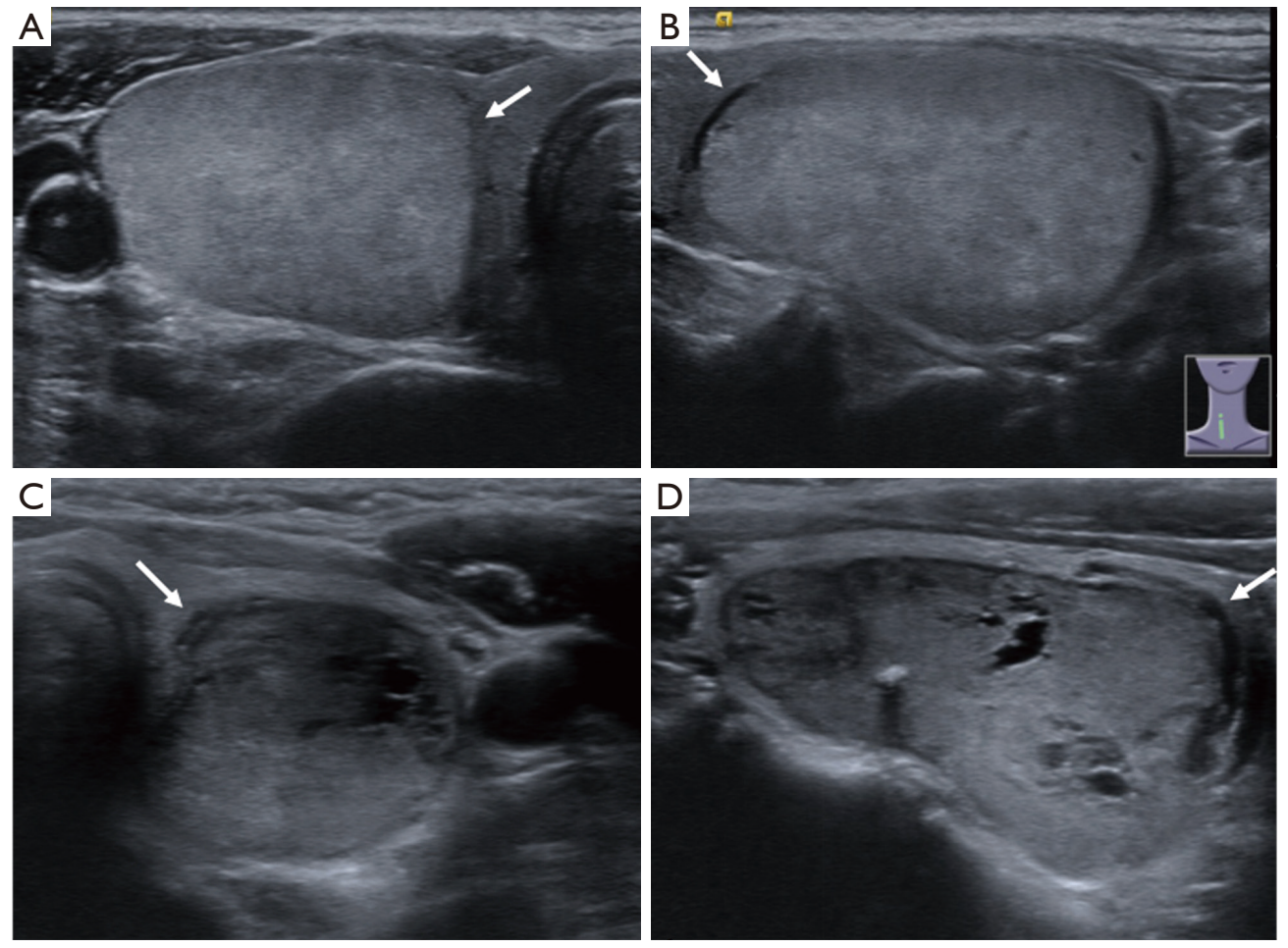

Figure 1 Conventional US images of follicular adenoma and follicular carcinoma. (A,B) A 61-year-old female patient with follicular adenoma. Gray-scale ultrasound image shows the transverse and longitudinal views of an isoechoic and slightly heterogeneous nodule with smooth halo (white arrow) in the right lobe of the thyroid. (C,D) A 52-year-old female patient with follicular carcinoma. Gray-scale ultrasound image shows the transverse and longitudinal views of a hypoechoic and heterogeneous nodule with calcification and an unevenlythick halo (white arrow) in the left lobe of the thyroid gland.

researchers found no significant difference in echotexture between follicular thyroid carcinoma and thyroid adenoma $(23,25)$. Our study obtained the results similar to the former: over $90 \%$ of follicular carcinomas had heterogeneous echotexture, while thyroid adenomas appeared more homogeneous in echotexture. Presumptively, there are both follicular structures and solid proliferation with rich cell components in thyroid follicular carcinomas, which might result in different echo intensity of acoustic reflection.

Peripheral halo sign is considered as a hypoechoic ring surrounding a thyroid nodule, and its role in differentiating thyroid follicular neoplasms is still under debate. Zhang et al. (23) reported that the thick and uneven halo around the nodule was more common in thyroid follicular carcinoma, but it was not an independent risk factor for thyroid follicular carcinoma. While Sillery et al. (22) thought the halo corresponded with the well-defined capsule that surrounds follicular adenomas, and the presence of a halo sign was more frequently associated with benign follicular neoplasia. In this study, unevenly thick halo was found to be more common in follicular carcinomas than adenomas, and it showed the highest diagnostic accuracy $(86.0 \%$ and $86.1 \%)$ as an independent risk factor for follicular carcinoma $(\mathrm{OR}=5.643,95 \%$ CI: 3.234-9.848; OR $=5.770,95 \%$ CI: $1.310-25.409)$ in both of the two multivariable logistic regression models. In addition, we found the halo sign was the representation of peripheral blood vessels in two-dimensional ultrasound in most cases (91/114 in FA, and 57/61 in FTC), which is consistent with the description of previous research (26); while in some other cases, color flow signal could not be detected from the halo, indicating that there might be other pathological changes for the formation of hypoechoic halo sign. Early studies have found follicular carcinomas generally have a well-defined, complete capsule with a thickness of $0.1-0.3 \mathrm{~cm}$, and the capsule is composed of parallel collagenous fibers, often containing medium-sized blood vessels with irregular thickness and edema of muscular walls 
Table 3 Contrast-enhanced ultrasound features of FTC and FA

\begin{tabular}{|c|c|c|c|}
\hline Feature & FTC $(n=25)$ & $\mathrm{FA}(\mathrm{n}=47)$ & $P$ value \\
\hline Arrival time & & & 0.379 \\
\hline Earlier & 17 & 26 & \\
\hline Synchronous & 4 & 15 & \\
\hline Later & 4 & 6 & \\
\hline Mode of entrance & & & 0.293 \\
\hline Centripetal & 16 & 24 & \\
\hline Non-centripetal & 9 & 23 & \\
\hline Peak intensity & & & 0.93 \\
\hline Hypo-enhancement & 6 & 9 & \\
\hline Iso-enhancement & 3 & 6 & \\
\hline Hyper-enhancement & 16 & 32 & \\
\hline Homogeneity & & & 0.554 \\
\hline Homogeneous & 13 & 21 & \\
\hline Heterogeneous & 12 & 26 & \\
\hline Early washout & & & 0.04 \\
\hline Absent & 6 & 23 & \\
\hline Present & 19 & 24 & \\
\hline Wash-out style & & & 0.017 \\
\hline Rapid & 10 & 7 & \\
\hline Slow & 15 & 40 & \\
\hline Perfusion defects & & & 0.264 \\
\hline Absent & 13 & 18 & \\
\hline Present & 12 & 29 & \\
\hline Range of perfusion defects & & & 0.674 \\
\hline$<50 \%$ & 24 & 44 & \\
\hline$\geq 50 \%$ & 1 & 3 & \\
\hline Rim enhancement & & & $<0.001$ \\
\hline Absent or irregular & 24 & 23 & \\
\hline Present & 1 & 24 & \\
\hline
\end{tabular}

FTC, follicular thyroid carcinoma; FA, follicular adenoma.

$(27,28)$, which may help explain why the capsule tends to be thicker and more irregular in follicular carcinomas than adenomas.

Calcifications (including microcalcifications, macrocalcifications and peripheral calcifications) are common ultrasound findings within thyroid nodules.
Actually, microcalcifications have been generally recognized as an important sonographic feature for differentiating between malignant and benign nodules especially in papillary carcinoma $(12,29)$. Nevertheless, the diagnostic value of calcification for follicular carcinoma is still unknown. In the present study, though we found the overall prevalence of calcification in the involved follicular neoplasms is at a low level, the presence of calcifications was more common in follicular carcinomas as an independent risk factor for follicular carcinoma $(\mathrm{OR}=1.748,95 \%$ CI: 1.098-2.783) in the multivariable logistic regression model of conventional US. This point is consistent with the results reported by Zhang and Kuo et al. (23,24). With regard to the subgroups of calcifications in this study, microcalcification was present in small numbers (12 in FA and 7 in FTC), while macrocalcifications and peripheral calcifications were more frequently found in FTC than FA. The presence of calcifications and calcification types in follicular carcinomas might indicate the internal state of metabolic disorder, thus a further larger study is needed to find the potential pathological correlation.

The aforementioned sonographic features including heterogenicity, unevenly thick halo and calcifications were all identified as independent risk factors for follicular carcinoma in our further multivariable analysis, and the AUC of the model used to determine the probability of malignancy was 0.835 with a $95 \%$ CI of $0.782-0.889$. In addition, on the basis of univariable analysis, several other sonographic features including margin, border, extracapsular extension, cystic changes, range of cystic changes, nodule vascularity differed significantly between FA and FTC. Indeed, predominantly solid, irregular margins, or chaotic arrangement of internal vascularity has been reported to be suggestive of FTC in previous studies, respectively $(22,23)$. Even so, none of these features in the present study were confirmed as significant risk factors for follicular carcinoma, and it remains unclear whether they could help differentiate the two types of follicular neoplasms.

To the best of our knowledge, our study is the first in the radiologic literature focusing on the diagnostic value of the contrast-enhanced features in the differentiation between thyroid follicular carcinoma and thyroid adenoma. Among the contrast-enhanced characteristics, our study showed early and rapid washout without rim enhancement were more frequently present in follicular carcinomas. When these significant CEUS factors were put into the further multivariable analysis along with other potential sonographic features, the final diagnostic model displayed 

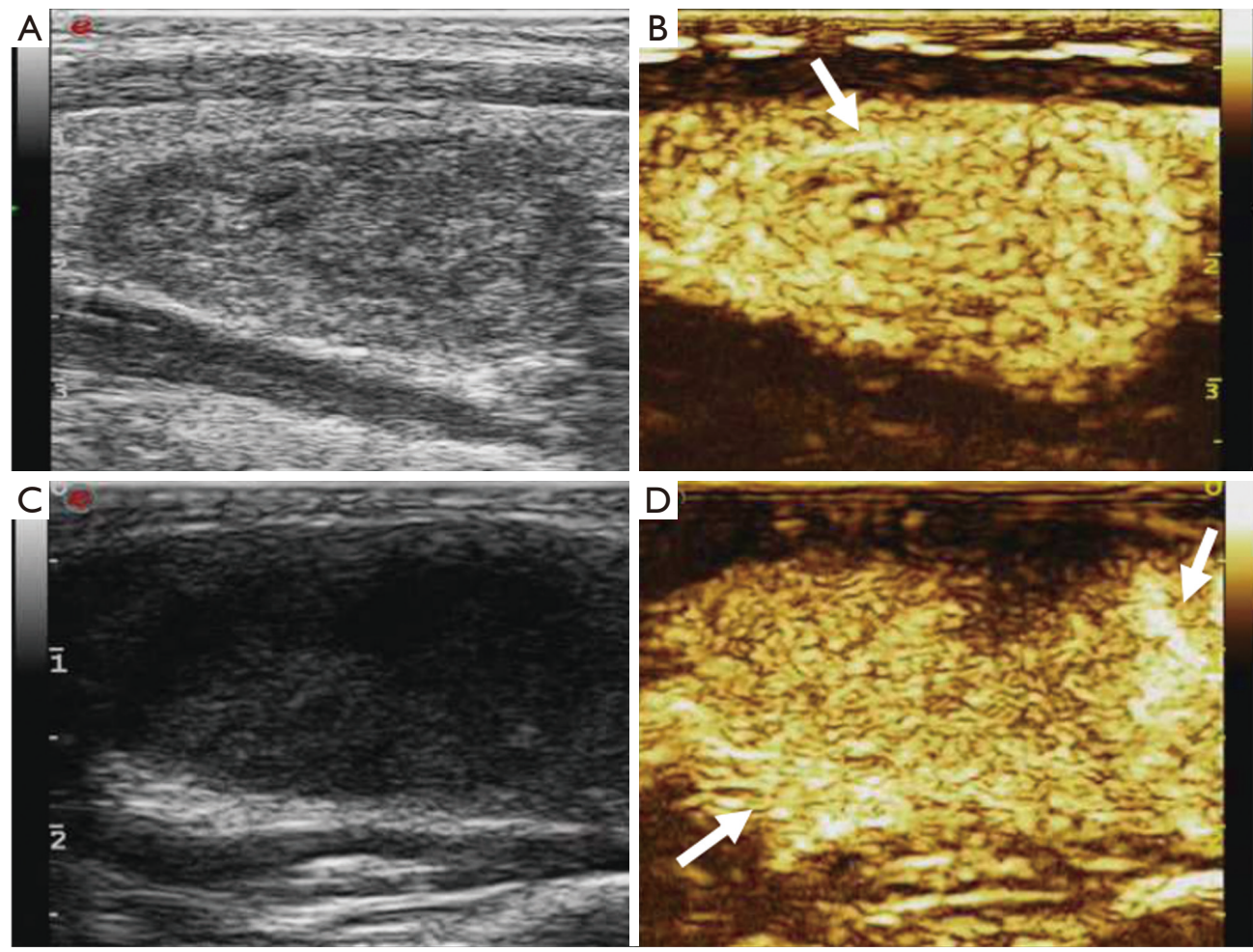

Figure 2 Conventional US and CEUS images of follicular adenoma and follicular carcinoma. (A,B) A 40-year-old male patient with follicular adenoma. Gray-scale and contrast-enhanced ultrasound image shows the longitudinal views of a hypoechoic and heterogeneous nodule with thin and regular rim enhancement (white arrow) in the right lobe of the thyroid. (C,D) A 43-year-old female patient with follicular carcinoma. Gray-scale and contrast-enhanced ultrasound image shows the longitudinal views of an extremely hypoechoic and heterogeneous nodule with irregular rim enhancement (white arrow) in the right lobe of the thyroid.

Table 4 Performance of CEUS and conventional US characteristics in diagnosis of follicular adenomas and carcinomas

\begin{tabular}{|c|c|c|c|c|c|}
\hline & Sensitivity, \% & Specificity, \% & PPV, \% & NPV, \% & Accuracy, \% \\
\hline \multicolumn{6}{|l|}{ Independent risk factors for model $1^{*}$} \\
\hline Heterogenicity & 91.9 & 48.3 & 47 & 92.2 & 62.8 \\
\hline Unevenly thick halo & 64 & 97.1 & 91.7 & 84.3 & 86 \\
\hline Calcifications & 36 & 81.4 & 49.2 & 71.8 & 66.3 \\
\hline Unevenly thick halo & 68 & 95.7 & 89.5 & 84.9 & 86.1 \\
\hline Absent or irregular rim enhancement & 96 & 51.1 & 51.1 & 96 & 66.7 \\
\hline
\end{tabular}

Model $1^{*}$ refers to the multivariate logistic regression model of conventional US, and model $2^{*}$ refers to the multivariate logistic regression model of CEUS in combination with conventional US. US, conventional ultrasound; CEUS, contrast-enhanced ultrasound; PPV, positive predictive value; NPV, negative predictive value.

good diagnostic performance with the AUC of 0.838 , and only absent or irregular rim enhancement $(\mathrm{OR}=27.000$, 95\% CI: 2.445-298.178) and unevenly thick halo (as aforesaid) were confirmed as independent risk factors for follicular carcinoma.

Ring enhancement has been reported as a valuable 

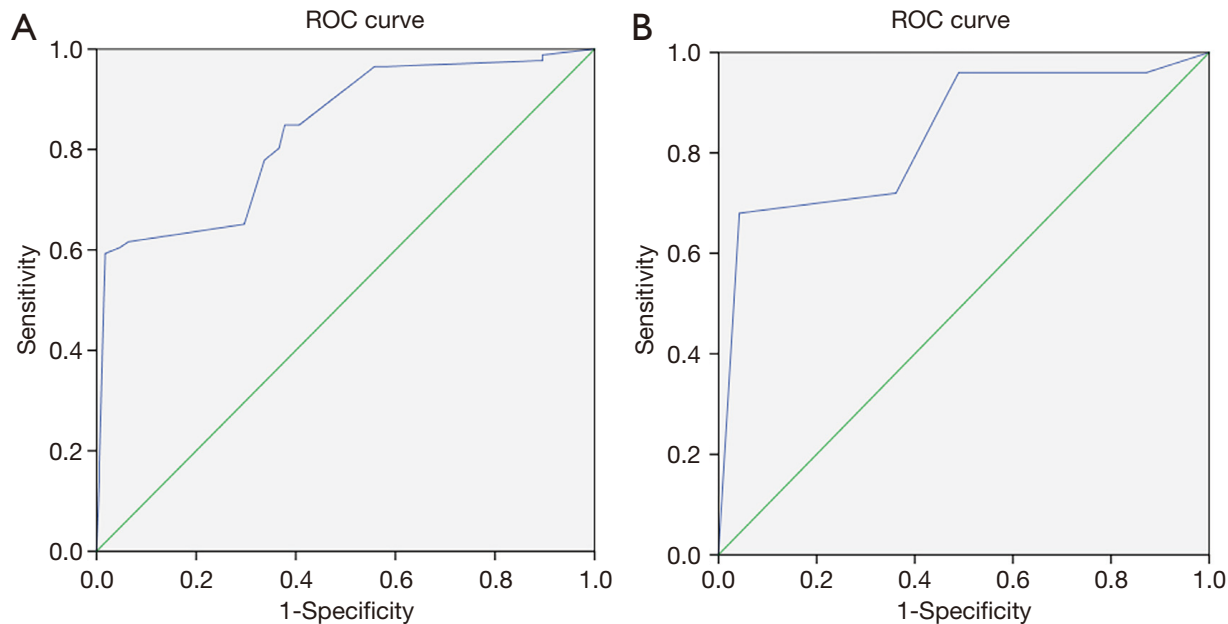

Figure 3 Receiver operating characteristic (ROC) curve of the final two logistic regression models for defining the preoperative diagnostic value of the factors in differentiating follicular adenomas from follicular carcinomas. The AUC (area under the curve) of multivariable logistic regression model of conventional ultrasound was 0.835 (A), while the AUC of multivariable logistic regression model of contrastenhanced US in combination with conventional ultrasound was 0.838 (B).

indicator for benign thyroid nodules with high sensitivity and specificity $(18,19)$, but its diagnostic role in the differentiation between thyroid follicular carcinoma and thyroid adenoma has not yet been discussed. Our study found that follicular adenoma usually exhibited thin and regular rim enhancement (may not be complete ring enhancement) on CEUS. Ring enhancement is considered to be associated with capsular and peripheral compressed parenchymal vessels around the lesion (18). Follicular adenoma often has a complete capsule, whereas capsular or vascular invasion, the typical feature of follicular carcinoma which refers to the penetration of the tumor into a vessel or capsule, might result in the absent or irregular rim enhancement.

Certainly, there are still several limitations in our study. First, the interpretive results may vary among different radiologists before operation. However, all preoperative sonograms of this study were interpreted again in random order on the screen by two experienced radiologists by consensus despite the lack of intra- and inter-observer reproducibility testing. Additionally, though early and rapid washout differ significantly in the univariable analysis, they didn't show much more significance in the further logistic regression analysis for the differential diagnosis of follicular neoplasms, and we speculate the reason for this may be related to the small sample. Indeed, because of the rarity of follicular carcinoma, the enrolled sample size in this study is rather small especially in the CEUS group. According to daily clinical practice, we usually make a diagnosis on the basis of all the significant sonographic features, and so the combined value of conventional US and CEUS, but not the value of CEUS alone, was deemed necessary for the analysis. In the current study, since AUCs for the diagnostic models including or excluding CEUS indicated similar diagnostic value (0.838 and 0.835 ), it showed that diagnostic models including or excluding CEUS both had high diagnostic value indeed in the differentiation between FTC and FA. On the other hand, it seemed that CEUS did not show additional diagnostic value to help distinguish follicular neoplasms, therefore larger study is required for further observations.

\section{Conclusions}

According to our study, follicular neoplasms with the following sonographic features including heterogenicity, unevenly thick halo and calcifications were considered highly suspicious as follicular carcinomas. While further CEUS imaging also supported the diagnosis by absent or irregular rim enhancement with good diagnostic value though it did not show additional diagnostic value to help distinguish follicular neoplasms in the current study. In conclusion, conventional US is an efficient diagnostic tool in the differential diagnosis of FTC and FA to help clinicians in making appropriate decisions while CEUS failed to provide additional diagnostic value in the study, 
thus the value of CEUS in the diagnosis of benign and malignant follicular neoplasms remains to be verified by further studies with larger sample sizes.

\section{Acknowledgments}

We would like to thank Mr Atte Lotjonen for his help in polishing our paper.

Funding: None.

\section{Footnote}

Reporting Checklist: The authors have completed the STARD reporting checklist. Available at https://dx.doi. org/10.21037/gs-21-535

Data Sharing Statement: Available at https://dx.doi. org/10.21037/gs-21-535

Conflicts of Interest: All authors have completed the ICMJE uniform disclosure form (available at https://dx.doi. org/10.21037/gs-21-535). The authors have no conflicts of interest to declare.

Ethical Statement: The authors are accountable for all aspects of the work in ensuring that questions related to the accuracy or integrity of any part of the work are appropriately investigated and resolved. The study was conducted in accordance with the Declaration of Helsinki (as revised in 2013). The study was approved by the institutional review board of Shanghai Jiao Tong University Affiliated Sixth People's Hospital [No. 2018-KY-040 (K)] and individual consent for this retrospective analysis was waived.

Open Access Statement: This is an Open Access article distributed in accordance with the Creative Commons Attribution-NonCommercial-NoDerivs 4.0 International License (CC BY-NC-ND 4.0), which permits the noncommercial replication and distribution of the article with the strict proviso that no changes or edits are made and the original work is properly cited (including links to both the formal publication through the relevant DOI and the license). See: https://creativecommons.org/licenses/by-nc-nd/4.0/.

\section{References}

1. Sobrinho-Simões M, Eloy C, Magalhães J, et al. Follicular thyroid carcinoma. Mod Pathol 2011;24 Suppl 2:S10-8.

2. Machens A, Holzhausen HJ, Dralle H. The prognostic value of primary tumor size in papillary and follicular thyroid carcinoma. Cancer 2005;103:2269-73.

3. Lumachi F, Borsato S, Tregnaghi A, et al. FNA cytology and frozen section examination in patients with follicular lesions of the thyroid gland. Anticancer Res 2009;29:5255-7.

4. Udelsman R, Westra WH, Donovan PI, et al. Randomized prospective evaluation of frozen-section analysis for follicular neoplasms of the thyroid. Ann Surg 2001;233:716-22.

5. Castro MR, Espiritu RP, Bahn RS, et al. Predictors of malignancy in patients with cytologically suspicious thyroid nodules. Thyroid 2011;21:1191-8.

6. Asa SL, Mete O. Thyroid neoplasms of follicular cell derivation: a simplified approach. Semin Diagn Pathol 2013;30:178-85.

7. Suster S. Thyroid tumors with a follicular growth pattern: problems in differential diagnosis. Arch Pathol Lab Med 2006;130:984-8.

8. Stolf BS, Santos MM, Simao DF, et al. Class distinction between follicular adenomas and follicular carcinomas of the thyroid gland on the basis of their signature expression. Cancer 2006;106:1891-900.

9. Smith J, Cheifetz RE, Schneidereit N, et al. Can cytology accurately predict benign follicular nodules? Am J Surg 2005;189:592-5; discussion 595.

10. Carpi A, Nicolini A, Gross MD, et al. Controversies in diagnostic approaches to the indeterminate follicular thyroid nodule. Biomed Pharmacother 2005;59:517-20.

11. Gharib H, Goellner JR. Fine-needle aspiration biopsy of the thyroid: an appraisal. Ann Intern Med 1993;118:282-9.

12. Haugen BR, Alexander EK, Bible KC, et al. 2015 American Thyroid Association Management Guidelines for Adult Patients with Thyroid Nodules and Differentiated Thyroid Cancer: The American Thyroid Association Guidelines Task Force on Thyroid Nodules and Differentiated Thyroid Cancer. Thyroid 2016;26:1-133.

13. Brito JP, Gionfriddo MR, Al Nofal A, et al. The accuracy of thyroid nodule ultrasound to predict thyroid cancer: systematic review and meta-analysis. J Clin Endocrinol Metab 2014;99:1253-63.

14. Frates MC, Benson CB, Charboneau JW, et al. Management of thyroid nodules detected at US: Society of Radiologists in Ultrasound consensus conference statement. Radiology 2005;237:794-800.

15. Kwak JY, Jung I, Baek JH, et al. Image reporting and 
characterization system for ultrasound features of thyroid nodules: multicentric Korean retrospective study. Korean J Radiol 2013;14:110-7.

16. Koike E, Noguchi S, Yamashita H, et al. Ultrasonographic characteristics of thyroid nodules: prediction of malignancy. Arch Surg 2001;136:334-7.

17. Bartolotta TV, Midiri M, Galia M, et al. Qualitative and quantitative evaluation of solitary thyroid nodules with contrast-enhanced ultrasound: initial results. Eur Radiol 2006;16:2234-41.

18. Zhang B, Jiang YX, Liu JB, et al. Utility of contrastenhanced ultrasound for evaluation of thyroid nodules. Thyroid 2010;20:51-7.

19. Ma JJ, Ding H, Xu BH, et al. Diagnostic performances of various gray-scale, color Doppler, and contrast-enhanced ultrasonography findings in predicting malignant thyroid nodules. Thyroid 2014;24:355-63.

20. Wu Q, Wang Y, Li Y, et al. Diagnostic value of contrastenhanced ultrasound in solid thyroid nodules with and without enhancement. Endocrine 2016;53:480-8.

21. Raber W, Kaserer K, Niederle B, et al. Risk factors for malignancy of thyroid nodules initially identified as follicular neoplasia by fine-needle aspiration: results of a prospective study of one hundred twenty patients. Thyroid 2000;10:709-12.

Cite this article as: Wu Q, Qu Y, Li Y, Liu Y, Shen J, Wang Y. Logistic regression analysis of contrast-enhanced ultrasound and conventional ultrasound of follicular thyroid carcinoma and follicular adenoma. Gland Surg 2021;10(10):2890-2900. doi: $10.21037 /$ gs-21-535
22. Sillery JC, Reading CC, Charboneau JW, et al. Thyroid follicular carcinoma: sonographic features of 50 cases. AJR Am J Roentgenol 2010;194:44-54.

23. Zhang JZ, Hu B. Sonographic features of thyroid follicular carcinoma in comparison with thyroid follicular adenoma. J Ultrasound Med 2014;33:221-7.

24. Kuo TC, Wu MH, Chen KY, et al. Ultrasonographic features for differentiating follicular thyroid carcinoma and follicular adenoma. Asian J Surg 2020;43:339-46.

25. Mihai R, Parker AJ, Roskell D, et al. One in four patients with follicular thyroid cytology (THY3) has a thyroid carcinoma. Thyroid 2009;19:33-7.

26. Clark KJ, Cronan JJ, Scola FH. Color Doppler sonography: anatomic and physiologic assessment of the thyroid. J Clin Ultrasound 1995;23:215-23.

27. Evans HL. Follicular neoplasms of the thyroid. A study of 44 cases followed for a minimum of 10 years, with emphasis on differential diagnosis. Cancer 1984;54:535-40.

28. Yamashina M. Follicular neoplasms of the thyroid. Total circumferential evaluation of the fibrous capsule. Am J Surg Pathol 1992;16:392-400.

29. Kwak JY, Han KH, Yoon JH, et al. Thyroid imaging reporting and data system for US features of nodules: a step in establishing better stratification of cancer risk. Radiology 2011;260:892-9. 